\title{
Presence of circulating Her-2 reactive CD8 T cells is associated with a lower frequency of MDSCs and better survival in elderly breast cancer patients
}

\author{
Jithendra Kini Bailur ${ }^{1 *}$, Brigitte Gueckel ${ }^{2}$, Graham Pawelec ${ }^{1}$, Evelyna Derhovanessian ${ }^{1}$ \\ From Society for Immunotherapy of Cancer 29th Annual Meeting \\ National Harbor, MD, USA. 6-9 November 2014
}

Breast cancer is one of the most common cancers among women. The risk of breast cancer has increased dramatically recently and a higher number of elderly women are being diagnosed with the disease. Myeloid-derived suppressor cells (MDSCs) have been implicated in breast cancer prognosis. Their frequency has been associated with tumour burden and studies have shown a poor prognosis in cases of metastatic breast cancer. MDSCs are known to impair the proliferation of $\mathrm{T}$ cells and to promote $\mathrm{T}$ cell apoptosis. Associations between MDSC levels and immune responses of the host to tumourassociated antigens had not been investigated. Here, we have studied $\mathrm{T}$ cell responses to Her-2 antigens, as well as the frequency of Tregs and MDSCs, in 40 untreated breast cancer patients (65-87 years of age) at diagnosis. After a 12-day in vitro expansion of memory cells stimulated by Her-2-derived peptides, CD4+ T cells reactive to Her-2 were detected in $84 \%$ of patients by intracytoplasmic staining simultaneously for TNF, IFN- $\gamma$, IL-2, IL-5, IL-10 and IL-17. In contrast, only $47 \%$ of patients had Her-2-reactive $\mathrm{CD} 8+\mathrm{T}$ cells. The patients who lacked a CD8 response tended to have higher frequencies of Lineage(neg) CD14+ HLA-DR(neg) cells $(p=0.09)$. Importantly, the 5 -year survival rate of patients who mounted a CD8 $+\mathrm{T}$ cell response and had a lower frequency of this particular subset of MDSC was $100 \%$ compared to only $53 \%$ in patients without Her-2-reactive CD8+ T cells and with higher frequencies of MDSCs $(\mathrm{p}=0.03)$. This survival advantage was also observed in non-metastatic patients, with only a $38 \%$ 5-year survival in patients without a CD8 response and high levels of MDSCs compared to 100\% survival of those who had a CD8 response and a lower frequency of MDSCs $(p=0.016)$. Similarly, for Tregs, patients who lacked a CD8 response to Her-2 and had higher frequencies of CD4+ Foxp3+ CD127(low) CD25+ Tregs had only $50 \%$ survival when compared to the $100 \%$ survival of the patients who mounted a CD8 response and had lower frequency of Tregs $(p=0.03)$. Also, for activated CD4+ CD45RA(neg)Foxp3(hi) Tregs, a similar trend was observed with $57 \%$ survival in patients who lacked a CD8 response and had higher frequencies of activated Tregs compared to the $100 \%$ survival in patients with a CD8 response and lower frequencies of activated Tregs $(p=0.06)$. Our data thus demonstrate a negative role of MDSCs and Tregs in prognosis of breast cancer patients, which might be through dampening favorable immune responses to tumour-associated antigens.

\section{Authors' details}

'Department of Internal Medicine II, Section for Transplantation Immunology and Immunohaematology, University Hospital, Tuebingen, Germany.

${ }^{2}$ Radiology Clinic, Diagnostic and Interventional Radiology, University Hospital, Tuebingen, Germany.

Published: 6 November 2014

doi:10.1186/2051-1426-2-S3-P151

Cite this article as: Kini Bailur et al:: Presence of circulating Her-2 reactive CD8 T cells is associated with a lower frequency of MDSCs and better survival in elderly breast cancer patients. Journal for ImmunoTherapy of Cancer 2014 2(Suppl 3):P151. 